\title{
LA CUANTIFICACIÓN DEL CONOCIMIENTO, 0 ¿QUÉ INVESTIGAR EN CONTADURÍA?
}

\section{Mario Ceballos Vásquez}

Tenía escrito parte de este trabajo cuando leí en un número anterior de Poliantea, el muy documentado artículo de Ignacio Vélez y Joseph Than sobre valoración de empresas ${ }^{1} \mathrm{y}$ volví a inquietarme sobre la forma de reflejar en los libros algunas de las realidades económicas. En efecto, para los contadores, los banqueros, los financieros y muchos otros profesionales es importante saber cuánto valen las empresas y más importante, si todos los elementos del valor de las empresas están presentes en los

\section{RESUMEN}

El valor de mercado de las empresas antes estaba en sus bienes tangibles. En la actual era del conocimiento está en sus activos intangibles, como su reputación, sus nombres de marcas o las ideas que tienen sus empleados. Sin embargo, los estados contables no reflejan adecuadamente lo que representan esos intangibles dentro del valor de las mismas.

En la medida en que Colombia avanza en el proceso de inserción en el mundo globalizado, las empresas se ven en riesgo de perder un patrimonio construido por muchos años con gran esfuerzo, por la enorme competencia que deben afrontar y que las pone en riesgo de desaparecer o de ser absorbidas, sin que se reconozca lo que vale el conocimiento que han acumulado. La universidad y las disciplinas de la Contaduría y la Administración de Empresas, harían un gran aporte si investigaran cuánto vale el factor conocimiento, cómo se calcula y cómo está reflejado en los estados financieros.

\section{PALABRAS CLAVE}

Medición del conocimiento, valor de mercado, medición del capital intelectual, Colombia, Facultades de economía, contaduría y administración.

\section{ABSTRACT}

The market value of enterprises was before in their tangible goods. At present, knowledge era is in its intangible assets, like reputation, trademark names or the ideas their employees have. However, the accounting statements don't reflect adequately what those intangibles reflect into their values themselves.

As long as in Colombia the insertion process advances in the globalized world, the enterprises take the risk of losing a built patrimony for many years with a great effort, due tot the enormous competence they must face and have the risk of disappearing or being absorbed without having recognition of the value they have accumulated. The university and the disciplines of Accounting and Business Administration would make a great contribution if they researched how much the knowledge factor is worth, how it is calculated and how it is reflected in the financing statements. 
El principal capital de una empresa no son las máquinasy los equipos, son las neuronas activables de su gente. Raymond Prada afirma que "cada persona, asu propia manera, agrega valor al mundo". estados contables. Mi preocupación más concreta es si el capital intelectual de las empresas está expresado en algún lugar de los estados financieros.

Para nadie debe ser un secreto que el mundo actual se mueve en torno al conocimiento, distribuido de forma dispareja, $y$ sobra decir, poco aclimatado en los países subdesarrollados. En las empresas, como en el mundo, se replica esta misma realidad. Unas con las más avanzadas tecnologías y otras apenas copiando adelantos del pasado. Vivimos en un nuevo contexto socioeconómico caracterizado fundamentalmente por la "producción, distribución y uso del factor conocimiento". Bajo este nuevo paradigma, los elementos intangibles están incrementando su importancia en detrimento de los factores de producción clásicos que hasta el momento habían sido definitorios de las economías más avanzadas; esto es: trabajo, maquinaria, instalaciones, etc. Así mismo, se afirma hoy que la creación de riqueza está más vinculada a los activos de índole intangible que a los activos materiales. El principal capital de una empresa no son las máquinas y los equipos, son las neuronas activables de su

\section{RESEÑA AUTOR:}

Mario Ceballos Vásquez, es economista de la Universidad Jorge Tadeo Lozano, con estudios en Contaduría de la Universidad Javeriana, y especialización en Docencia Universitaria de la Universidad Militar Nueva Granada. Durante cerca de treinta años colaboró en los bancos Cafetero, Exterior de los Andes y de España y Mercantil de Colombia, en las áreas financiera y de auditoría, al tiempo que, desde el año de 1984, se desempeñó como profesor de la Facultad de Administración de Empresas del Politécnico Grancolombiano, en el programa de Tecnología de Administración Bancaria. Actualmente está dedicado de tiempo completo al ejercicio docente y adelanta una maestría en docencia en la Universidad de la Salle. gente. Raymond Prada afirma que "cada persona, a su propia manera, agrega valor al mundo"2.

En un entorno cada día más dinámico, intangibles como las capacidades y formación de los recursos humanos, de los que se dice son la ventaja comparativa de las empresas, y la imagen corporativa, la estructura organizativa o las relaciones con clientes y proveedores, se están convirtiendo en importantes fuentes de ventajas competitivas sostenibles y de creación de valor agregado. Paradójicamente, estos elementos críticos para el éxito empresarial no están incluidos en los estados financieros de las compañías y, en muchas ocasiones, ni siquiera se cuenta con un método que permita identificarlos, medirlos y gestionarlos correctamente.

Este mayor énfasis en las personas y en sus aportes a las empresas, se contrapone al hecho de que las herramientas utilizadas para la medición de ese capital intelectual se manifiestan incapaces de incluir estos nuevos elementos y ofrecer explicaciones convincentes sobre fenómenos como la supervivencia, el crecimiento, la productividad o el empleo. Vale decir, que la medición se ha convertido en una necesidad estratégica de nuestros días y en consecuencia, es crucial la construcción de nuevos sistemas e indicadores de medición que capturen y reflejen la realidad actual en toda su dimensión.

Para remediarlo, en el mundo se están haciendo notables esfuerzos desde el ámbito académico y empresarial para dar forma a un modelo de carácter general que permita a las empresas incluir los intangibles en sus sistemas de gestión internos y en sus forma- 
tos de difusión de información. Este tipo de mediciones son del campo de disciplinas como la Contaduría o la Administración.

\section{PERO ¿QUÉES CONOCIMIENTO EN EL MUNDO EMPRESARIAL?}

Según Polanyi, citado por Bueno ${ }^{3}$, existen dos tipos de conocimiento:

- El conocimiento explícito, entendiendo como tal, un conocimiento codificado, sistemático y transferible a través del lenguaje formal.

- El conocimiento tácito, es decir, un conocimiento personal, implícito, difícil de formalizar, de medir y, por tanto, de comunicar ${ }^{4}$.

Otros tratadistas a la hora de analizar el conocimiento establecen una distinción en cuatro categorías del saber: qué, por qué, cómo y quién:

- Saber qué (know what). Se refiere a hechos. El conocimiento en esta categoría esta más cerca de asemejarse al concepto de información.

- Saber por qué (know why). Se refiere al conocimiento científico de los principios y leyes de la naturaleza. La producción y reproducción de este tipo de conocimiento está frecuentemente centrada en organizaciones especializadas, como laboratorios de investigación y universidades. Para tener acceso al mismo las empresas necesitan de interacciones con estas organizaciones, ya sea reclutando personal cualificado o bien mediante contactos y actividades conjuntas.
- Saber cómo (know how). El más conocido. Esta categoría incluye las habilidades y capacidades para hacer algo. Una de las razones más importantes para la formación de redes industriales es la necesidad de ser capaz de compartir y combinar elementos del know-how.

- Saber quién (know who). Este tipo de conocimiento esta siendo cada vez más importante. Se refiere a la información sobre quién conoce qué y quién sabe cómo hacer algo. Se trata de conocimiento interno de la organización en un nivel mayor que cualquiera de las categorías anteriores. Los gerentes de empresa modernos consideran vital emplear este tipo de conocimiento como respuesta a la aceleración del cambio.

Los dos primeros, el saber qué y el saber por qué, se pueden obtener de manera cada día más sencilla a través de la lectura de libros, asistiendo a seminarios, conferencias y cursos de actualización, accediendo a bases de datos, internet, etc., pero los dos últimos, el saber cómo y el saber quién, se relacionan más con la experiencia práctica y forman parte del conocimiento tácito.

El concepto de conocimiento es mucho más amplio que el de información; se restringe éste último básicamente a las categorías de saber qué y saber por qué. El desarrollo de las tecnologías de la información puede ser considerado como una respuesta a la necesidad de las organizaciones de apoderarse del know what y del know why de los empleados de una manera más eficiente.

3 BUENO, E. SALMADOR, M. P. (2003): "La dirección del conocimiento en el proceso estratégico emergente de la empresa: información, complejidad e imaginación en la espiral del conocimiento". Madrid: Revista de Economía y Empresa, no 48, vol. XIX (2a época), pp. 17-32

4 Podría agregarse aquí otro tipo relacionado con las personas llamado el conocimiento "desconocido", del que no somos conscientes ( $\mathrm{y}$ al que se llega mediante la intuición), pero no es este el lugar para este tema. 
Las teorías evolutivas de la innovación y el cambio tecnológico no se limitan a explicar el crecimiento, sino que extienden su interés a los procesos de cambio, innovación y progreso técnico.
La nueva economía se caracteriza por la necesidad de un aprendizaje continuo, una constante actualización, tanto en lo que se refiere a información codificada como en lo relativo a las competencias necesarias para saber emplear y gestionar esta información, habilidades que se han convertido en cruciales debido a que el acceso a la información se está volviendo cada día más sencillo y barato gracias a tecnologías como el internet.

Por eso, información y conocimiento no pueden considerarse sinónimos. Es decir, aunque los desarrollos tecnológicos han incrementado y facilitado el interés por el conocimiento y su gestión, y la existencia de tecnologías de la información y las infraestructuras para las comunicaciones den un fuerte empuje al proceso de codificación de ciertos tipos de conocimiento, no pueden confundirse ambos conceptos.

Muchos afirman que actualmente estamos ante un nuevo paradigma socioeconómico, en lo que el conocimiento ha pasado a ser uno de los factores productivos determinantes, dejando en posiciones marginales a los factores de producción tradicionales: tierra, trabajo y capital.

Al desagregar los factores que intervienen en el crecimiento, algunos estudios han concluido que el factor conocimiento representa el $34 \%$ y el factor trabajo el $25 \%$, es decir, entre los dos representan el 59\%.

La teoría económica ha ponderado la relevancia de dos factores esenciales para comprender el desarrollo económico: los recursos humanos y la investigación y el desarrollo.
Por otro lado, la teoría de la empresa se ha centrado esencialmente en la organización empresarial del trabajo y la producción, los círculos de calidad, la mercadotécnia o la formación de los recursos humanos.

Finalmente, y en contraposición de las teorías anteriores, las teorías evolutivas de la innovación y el cambio tecnológico no se limitan a explicar el crecimiento, sino que extienden su interés a los procesos de cambio, innovación y progreso técnico ${ }^{5}$. Dichas teorías establecen que la actividad científica y tecnológica no es un factor exógeno, sino que estas se introducen en el modelo como una variable endógena explicativa del crecimiento económico. En palabras de Prada:

Una estadística de comercio de los Estados Unidos nos muestra que en ese país dos de cada diez empresas no alcanzan a estar activas como para celebrar su quinto aniversario. En algunos países de Latinoamérica la situación es aún peor, pues allí nueve de cada diez empresas no llega a su quinto aniversario. Las investigaciones sobre las causas de estos fracasos se asocian con la calidad deficiente de la idea de producto seleccionada y con una expectativa irrealista en sus planes de desarrollo. Esto nos indica que las razones atribuibles a la creatividad y a la planeación revisten más importancia que los motivos puramente financieros,... ${ }^{6}$

De manera gráfica, la figura 1 pone de manifiesto la evolución que los cuatro factores económicos principales han sufrido a lo largo del tiempo. En una eco-

5 CARDONA A. M.et al. Grupo de estudios sectoriales y territoriales, Departamento de Economía, Escuela de Administración - Universidad Eafit. "Diferencias y similitudes en las teorías del crecimiento económico". Ed. Eumed.net Págs. 44 - 65. Disponible en internet [07-05-05] ISBN 84-688-9043-X.

6 PRADA, Raymond. Creatividad innovación empresarial. Tecno Press Ediciones, 2003, p XIV. 
nomía agrícola, los recursos económicos más importantes eran la tierra y el trabajo; en la Era Industrial, el factor trabajo continuó siendo de gran relevancia y la importancia del capital aumentó en detrimento del factor tierra. Por último, en la Era del Conocimiento (la era actual), asistimos a un cambio radical en el protagonismo de los factores productivos tradicionales en el que al conocimiento se le otorga el papel más destacado. Se afirma, que el conocimiento es el motor de esta nueva economía.

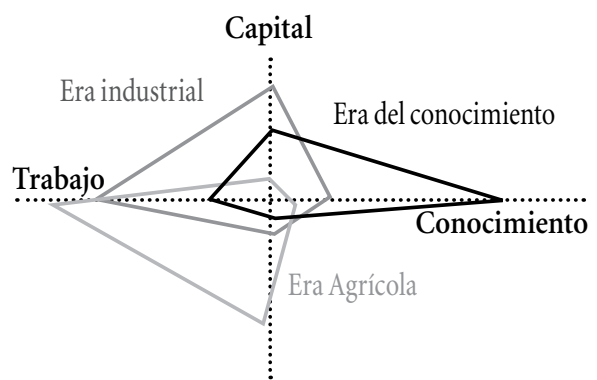

Tierra

Figura 1. Evolución de la Economía Fuente: Gorey y Dobat (1996) ${ }^{7}$

\section{Y ¿CÓMO ESTÁ NUESTRO PAÍS?}

Puede afirmarse que Colombia está desparramada en diferentes mundos, pues como lo afirman Jean-Michel Blanquer y Christian Gros en el libro Las dos Colombias ${ }^{8}$ :

En 1970, hace apenas algo más de treinta años, Colombia, con veintiún millones de habitantes, era un país relativamente poco poblado y todavía bastante rural (43\% de la población vivía en el campo) [¿Economía agrícola?]
Veinticinco años después, la población del país había aumentado enormemente -al alcanzar treinta y cinco millones de habitantes sobrepasó, en ese aspecto, a Argentina- y había cumplido ampliamente sus expectativas de desarrollo económico. El producto interno bruto había aumentado a más del doble (se multiplicó por 2,5) y su crecimiento nunca se interrumpió ni siquiera en los años ochenta, época negra para América Latina, el decenio perdido. Colombia sobrepasó al Perú y se acercó sensiblemente a su fraterno enemigo, Venezuela [¿Era Industrial?].

No obstante hoy subsisten en el país grandes desequilibrios de desarrollo entre sus diferentes regiones y la disparidad de ingresos coloca a Bogotá como la que tiene la mayor distorsión entre 71 ciudades de todos los continentes. (La diferencia entre el ingreso mayor y el ingreso menor en Oslo no alcanza a ser de dos veces y en Bogotá es de más de ocho) ${ }^{9}$.

Desde la segunda mitad del siglo pasado las principales teorías económicas reconocen, de un modo u otro, la existencia de elementos de carácter no tangible, y distintos a los factores tradicionales, que explican en gran medida el crecimiento económico. Es decir, la existencia de intangibles, entendiendo como tales aquellos factores que sin tener sustancia física ni material generan o pueden generar valor agregado y crecimiento económico. Bajo este amplio concepto encontramos cuestiones tales como la investigación y el desarrollo, la formación 
de la mano de obra, el software, el mercadeo, la capacidad de aprendizaje, la experiencia técnica o la estructura organizativa.

Los intangibles, por tanto, no constituyen un fenómeno nuevo. Lo que ha llevado a la reciente relevancia de los mismos se debe a la combinación de dos fuerzas, una de carácter socioeconómico y otra de carácter tecnológico. Por un lado, el incremento de la competencia en el mundo empresarial como consecuencia de los procesos simultáneos de globalización y liberalización de los sectores económicos clave (transportes, electricidad, telecomunicaciones, etc.) y, por otro, el desarrollo de las tecnologías de la información y comunicación que han posibilitado enormes cambios en la estructura y funcionamiento de las organizaciones, comparables, según la Unión Europea, a las revoluciones industriales de los siglos XVIII y XIX ${ }^{10}$.

Podemos decir, en consecuencia, que la economía moderna se caracteriza por tres elementos nuevos que la hacen radicalmente distinta a la de etapas anteriores ${ }^{11}$ : 1) El ritmo al que se producen cambios estructurales parece haberse incrementado en las ultimas tres décadas, lo que implica la necesidad de una mayor rapidez de respuesta y adaptación, 2) los cambios ocurren de manera simultánea, y 3 ) la idea de que el conocimiento explica el crecimiento parece ser de un orden de magnitud distinta de lo que ha sido históricamente.

Actualmente, y según los datos publicados por la OCDE 12 en 1996, se estima que más del 50\% del PIB de las mayores economías del mundo están basadas en conocimiento. Hay que destacar, por un lado, que las inversiones directamente relacionadas con productos y servicios de alta tecnología, particularmente con información y comunicaciones, se están incrementado. $Y$, por otro, que la demanda de empleo se caracteriza, de manera sensiblemente creciente, por ser altamente cualificada, fundamentalmente en los sectores relacionados con tecnología de punta.

Los datos publicados por esa organización demuestran que, efectivamente, el paradigma económico está cambiando, y con ello las fuentes de creación de valor y de competitividad para las empresas. Cada vez son más numerosas las voces en la bibliografía económica y en la gestión empresarial que tratan de destacar la verdadera importancia de los activos de carácter intangible en el éxito y el mantenimiento de las organizaciones en el mercado. En el mundo desarrollado, el proceso de competencia es más amplio que la mera decisión de fijación de precios y de niveles de producción. Ya no es suficiente la adquisición de una planta o una maquinaria nueva más avanzada, por la sencillez con la que puede ser plagiada por el mercado. Al lado, las inversiones en intangibles (gestión, formación, mercadeo, innovación...) son más difíciles de percibir y, por tanto, de copiar por los competidores. Es decir, la capacidad de adaptación al cambio, así como la mejora continua, se han convertido en requisitos

10 COMISIÓN EUROPEA, Hacia la Europa basada en el conocimiento. La Unión Europea y la sociedad de la información. Disponible en internet: http://europa.eu.int/comm/publications/booklets/move/36/index_ es.htm [31-05-05].

11 COMISIÓN EUROPEA, Op. Cit.

12 Organización para la Cooperación y el Desarrollo Económico, organización con 30 países miembros, y 70 países o economías en desarrollo y transición asociadas con su trabajo. 
básicos para alcanzar ventajas competitivas en este nuevo entorno.

Dentro de este contexto nuestro país evidencia el atraso de su economía con las cifras que trae el informe de la Misión de Ciencia, Educación y Desarrollo, "Colombia: Al filo de la oportunidad"13, cuando muestra que en Colombia la inversión en educación como porcentaje del PIB era apenas del 2,80\% en 1987 y la inversión en Ciencia y Tecnología del 0,2\% en el mismo año.

Por todo lo anterior la medición del conocimiento se ha convertido en un elemento crucial para poder establecer comparaciones de nuestras empresas con las del resto del mundo y de nuestra economía desde un punto de vista macroeconómico. A partir de la Segunda Guerra Mundial indicadores tales como el PIB y las cuentas nacionales se articularon como los indicadores económicos estándares para medir en cifras agregadas, fenómenos como la producción, el consumo, la inversión o el empleo. Estos índices económicos permitían vislumbrar cómo se comportaba o funcionaba un sistema y sirven como guía para la toma de decisiones en el ámbito político, social y económico de todos los agentes del sistema, pero nunca han podido explicar o predecir el desarrollo económico de una nación, entre otros, porque no han sido capaces de dar un paso más allá de mostrar el valor agregado de bienes y servicios ni de incluir determinadas variables que también son representativas de la realidad económica y social ${ }^{14}$.

Ante la insatisfacción provocada por las explicaciones proporcionadas por las teorías neoclásicas del crecimiento y por el PIB como indicador válido y único de bienestar social, algunos economistas han intentado buscar otros indicadores que fueran capaces de reflejar en mayor medida la enorme complejidad que caracteriza la realidad social. En este sentido, se han hecho diversos aportes, entre las que cabe destacar:

1. La inclusión de ajustes en la medida del PIB existente, como por ejemplo, introduciendo variables medioambientales, dando lugar al denominado PIB Verde.

2. La sustitución del PIB por otro indicador diseñado de forma completamente distinta.

3. La utilización de cuentas satélite.

4. El desarrollo de los denominados "indicadores sociales"15, que suponen un complemento al PIB tradicional. Uno de esos indicadores es el Índice de Desarrollo Humano ${ }^{16}$ que combina PIB per cápita, tasa de alfabetización de adultos y tasa bruta combinada de matriculación primaria, secundaria y superior.

Todo ello pone de manifiesto la existencia de una importante carencia que dificulta la comprensión de cómo funcionan realmente los engranajes de esta nueva economía, y que supone un inquietante reto para las investigaciones presentes y futuras dedicadas al diseño de indicadores e instru-
La medición del conocimiento se ha convertido en un elemento crucial para poder establecer comparaciones de nuestras empresascon las del resto del mundo $y$ de nuestra economía desde un punto de vista macroeconómico.

13 Misión de Ciencia, Educación y Desarrollo. “Colombia: Al filo de la oportunidad”.Bogotá. Colección Mesa Redonda, Cooperativa Editorial Magisterio, 1995, Varios autores.

14 Variables tales como el trabajo de las amas de casa o el costo medioambiental en términos de destrucción de la capa de ozono, polución, etc.

15 Índice de Desarrollo Humano (IDH), Índice de Desarrollo Relativo al Género (IDG) y el Índice de Potenciación de Género (IPG), e Índice de Pobreza Humana (IPH).

16 Colombia aparece en el puesto 64 entre los países considerados. 
Una política de investigación $y$ desarrollo $(I+D)$ exitosa parte de ideas que provienen de fuentes diversas, incluyendo intercambios profesionales informales $y$ experiencias $y$ sugerencias de usuarios. mentos de medición fiables y acordes con este nuevo paradigma socioeconómico, no solo en el ámbito empresarial, sino también en el ámbito macroeconómico.

La OCDE afirma que, al no ser el conocimiento una entrada (input) económica tradicional, como pueden ser las materias primas o el trabajo, la inclusión en las cuentas nacionales tradicionales es algo problemático, pues cambiaría la propia forma de cálculo de estos indicadores. Por todo ello, dicha institución argumenta cuatro razones por las cuales los indicadores de conocimiento no pueden aproximarse al alcance de los indicadores económicos clásicos:

- No existe una fórmula estable o "receta" por la cual traducir las entradas (inputs) de creación de conocimiento en salida (output) de conocimiento.

- Los ingresos de creación de conocimiento son difíciles de establecer porque no hay cuentas de conocimiento análogas a las cuentas nacionales tradicionales.

- El conocimiento carece de un sistema de precios sistemático que pudiera servir como base.

- Por último, la obsolescencia de unidades de inventario (stocks) de conocimiento no está documentada.

El problema de desarrollar nuevos indicadores es en sí mismo un elemento que pone de relieve el carácter único y diferente de la economía basada en el conocimiento. Para entender plenamente su funcionamiento se requieren nuevos conceptos y medidas que van más allá de las transacciones de mercado convencionales. Según este organismo, resulta, por tanto, necesario mejorar los indicadores de la economía basada en el conocimiento para desarrollar las siguientes tareas:

- Medir las entradas (inputs) de conocimiento. Tradicionalmente, los indicadores de gasto en investigación y desarrollo y los que hacen referencia al personal investigador y técnico han sido las mejores fuentes de información para intentar medir las entradas (inputs) del conocimiento. No obstante, tan solo una pequeña fracción de todas las entradas (inputs) de creación de conocimiento se pueden atribuir a éstos. En este sentido, la OCDE señala que "una política de investigación y desarrollo (I+D) exitosa parte de ideas que provienen de fuentes diversas, incluyendo intercambios profesionales informales $y$ experiencias y sugerencias de usuarios ${ }^{17}$." De este modo, la mejora en el proceso de recogida de datos resulta fundamental a la hora de reconocer la importancia relativa de las diversas entradas de conocimiento.

- Medir los inventarios (stocks) y los flujos del conocimiento. La medición y valoración de cualquier inveantario (stocks) lleva asociada la inclusión de un factor de depreciación en el tiempo. Por ello, resulta muy difícil aplicar criterios de amortización sobre indicadores de conocimiento. En relación con este problema, se plantea la posibilidad de acumular entradas (inputs) de I+D anuales para varios países e industrias y amortizarlos utilizando tasas de depreciación aproximadas.

Por otra parte, la medición de flujos o "la proporción de inventarios (stock) de conocimiento que entra en la economía en un 
periodo de tiempo determinado ${ }^{18}$ " resultan todavía más complicadas. Las variables proxy más utilizadas para medir flujos de conocimiento son: 1. Difusión incorporada (embodied difusión), que se refiere a la introducción en los procesos de producción de maquinaria, equipos y componentes que involucran tecnologías nuevas.

\section{Difusión no incorporada (disembo-} died difusión), referida a la transmisión de conocimiento, habilidades técnicas o tecnología en forma de patentes, licencias o saber cómo (know-how).

- Medir las salidas (outputs) del conocimiento. La puesta en relación entre las entradas (inputs) y las salidas (outputs) del conocimiento permite dar una idea de las posiciones relativas de los diversos sectores y países en términos de su intensidad en I+D. De este modo, la clasificación de la OCDE ordena sectores e industrias en relación con su gasto relativo en $\mathrm{I}+\mathrm{D}$ o a su intensidad en I+D. No obstante, este tipo de indicadores se utilizan principalmente en el sector manufacturero y no han sido desarrollados para el sector servicios, por lo que resulta necesario un mayor esfuerzo en esta dirección.

El cálculo de los costos y beneficios de la innovación para valorar las tasas de retorno social y privado es otra metodología que se utiliza para medir las salidas (outputs) del conocimiento. Este tipo de indicador ofrece, entre otros, una idea del beneficio neto del esfuerzo innovador en industrias y países; recientemente tam- bién se está construyendo en el ámbito de empresa.

- Medir las redes de conocimiento. La distribución del conocimiento entre actores, así como los cauces formales e informales por los que éste se transfiere, resulta fundamental a la hora de comprender el proceso de innovación y creación de conocimiento. El desarrollo de indicadores capaces de medir estas redes se basan, en la actualidad, en el Sistema Nacional de Innovación, que pretende dar una visión de la "habilidad de países y sistemas para distribuir conocimiento entre los diversos actores e instituciones"19, basándose en encuestas de innovación. A partir de estas encuestas se está comenzando a trabajar en mapas de sistemas nacionales de innovación y en la capacidad de distribución del conocimiento de las economías a través del análisis de dos flujos principales: 1. La distribución del conocimiento entre universidades, institutos públicos de investigación e industrias. 2. La distribución de conocimiento dentro del mercado entre proveedores y usuarios ${ }^{20}$.

- Medir aprendizaje y conocimiento. El desarrollo de indicadores que midan la relación entre aprendizaje y conocimiento resulta de enorme importancia tanto para países como para empresas. Las aproximaciones más comunes a estos indicadores se basan en la valoración de las tasas de retribución social o privada (social or private rates of return); esto es, en la medición del impacto que 
el gasto en educación en la sociedad y la formación empresarial tienen sobre el crecimiento económico o sobre los resultados empresariales, respectivamente.

Es necesario entonces que los nuevos indicadores registren el proceso de innovación y distribución del conocimiento entre los actores e instituciones claves en la economía.

La empresa, concebida como "caja negra" por las teorías económicas clásicas, está siendo ahora estudiada por nuevas disciplinas que prestan especial atención a las dinámicas interiores de esa "caja", al conocimiento embebido en las rutinas, prácticas y procesos que la compañía transforma en productos y servicios con valor agregado para el consumidor.

Sin embargo, y a pesar del relativo consenso existente sobre la relevancia de los recursos intangibles, la realidad contable no refleja esta creciente importancia y no se cuenta con sistemas de medición adecuados. Actualmente, diversas investigaciones, en las que se destaca el estudio citado con una muestra de las 500 mayores empresas de EE.UU., ponen de manifiesto que el valor medio de mercado de una compañía es normalmente de dos a nueve veces el valor reflejado en sus libros. Como demuestra la evidencia empírica, las cinco empresas con mayor cotización en la Bolsa de Nueva York presentan un valor de mercado muchas veces superior al que reflejan sus estados contables.

El problema fundamental a la hora de contabilizar dichos intangibles radica en el reconocimiento de los mismos como activos. Algunas de las limitaciones que se presentan a la hora de incluir determinada información en el sistema contable se deben a la propia estructura de la contabilidad. Las objeciones más importantes para su reconocimiento en la práctica emanan de la incertidumbre asociada a los beneficios de estos intangibles y al hecho de que, en algunos casos, la empresa no puede ejercer un control total sobre los mismos (como por ejemplo sobre el saber cómo know-how no patentado).

Adicionalmente, hay que tener en cuenta que la inmensa bibliografía que este campo de investigación está generando no sólo apunta que existen importantes deficiencias en la contabilización y publicación de información sobre intangibles que debieran ser subsanadas, si no que éstos no están integrados correctamente en los sistemas de gestión de las empresas. A pesar de ello, sí puede afirmase que son considerados de manera informal en dichos procesos de decisión y gestión. Este hecho constata la importancia que la empresa otorga a los mismos para sus objetivos de gestión interna. Sin embargo, y aunque efectivamente los directivos emplean indicadores de carácter no financiero en su gestión diaria, no disponen de buenos sistemas ni aplicaciones para medir y valorar los intangibles críticos de sus compañías. Por todo ello, resulta de vital importancia desarrollar un sistema o instrumento de medición del capital intelectual adecuado que facilite su identificación y medición, y posibilite una sistematización en el tiempo.

Los objetivos prioritarios que debiera cumplir este sistema de medición son, por un lado, el incremento de la competitividad de la compañía y, por otro, la mejora en la efectividad de su gestión interna.

La clave para llegar a una mejora sustancial de los sistemas de gestión y difusión de información sobre intangibles debe ser la 
construcción de una estructura comprensible y coherente de información que se centre en el proceso de creación de valor de la compañía.

Colombia y las facultades de Contaduría y Administración enfrentan un enorme reto que supone la incorporación del país a los tratados del ALCA y de libre comercio con los Estados Unidos, los cuales expondrán un capital hecho con mucho esfuerzo por los empresarios colombianos y extranjeros en el país a una enorme competencia y confrontación con las empresas de los demás países que se incorporarán a estos tratados. Si es cierto que estamos muy lejos de los de mayor desarrollo inclusive de América Latina, hay acervo cultural, de mercado, de conocimiento que debe preservarse y es mucho lo que la universidad puede y debe hacer a este respecto.

\section{BIBLIOGRAFÍA}

- BLANQUER, Jean-Michel, GROS, Christian (2002). Las dos Colombias. Bogotá: Editorial Norma.

- BOUNFOUR, A. (2003). The Management of Intangibles, the Organisation most Valuable Assets. Londres: Routledge.

- BUENO,E. (2005): Una reflexión crítica sobre la comprensión de la Sociedad y Economía del Conocimiento: la era de los intangibles". Madrid: Capital Intelectual. No 1, pp. 6-17.

- _________(2002): "El capital social en el nuevo enfoque del capital intelectual de las organizaciones". Revista de Psicología del Trabajo y de las Organizaciones, Vol. 18, No 32, pp. 157-176.

(1998): "El capital intangible como clave estratégica en la competencia actual". Boletín de Estudios Económicos, LIII, agosto, 164, pp. 205-229.

- BUENO, E. y SALMADOR, M. P. (2003): "La dirección del conocimiento en el proceso estratégico emergente de la empresa: información, complejidad e imaginación en la espiral del conocimiento". Revista de Economía y Empresa, no 48, vol. XIX (2a época), pp. 17-32.

- CARDONA, A. M. et al. Diferencias y similitudes en las teorías del crecimiento económico. Grupo de Estudios Sectoriales y Territoriales. Departamento de Economía, Escuela de Administración, Universidad Eafit. Ediciones Eumed.net Pág 52 - 65 .

- Clement, W., HAMmerer, G. y SCHWARZ, K. (1998). Measuring Intangible Investment, Intangible Investment from an Evolutionary Perspective, OCDE.

- Misión de CIENCIA, EDUCACIÓN y DESARROLLO (1995). Colombia: Al filo de la oportunidad. Bogotá. Colección Mesa Redonda, Cooperativa Editorial Magisterio.

- PRADA, Raymond (2003). Creatividad e Innovación Empresarial. Bogotá: Tecno Press Ediciones Ltda.

- SERRANO, C. (2005). "La Contabilidad en la era del conocimiento". [en línea] 5 campus.org, Sistemas Informativos Contables <http://www.5campus.org/ leccion/introduc $>$ [07-05-05]

- UNIÓN DE BANCOS SUIZOS (2003). Prices and Earnings. Comisión Europea, 2004.

- VÉLEZ Pareja Ignacio y THAN Joseph. “ ¿Coinciden EVA y Utilidad Económica (UE) con los métodos de flujo de caja descontado en valoración de empresas?”. Bogotá: Poliantea, No. 1, mayo, 2004. pp. $89-108$. 